\title{
Public health nursing interventions to promote quality of life in older adult populations: A systematic review
}

\author{
Marjorie A. Schaffer ${ }^{1}$, Mary Kalfoss ${ }^{2}$, Kari Glavin *2 \\ ${ }^{1}$ Bethel University, St. Paul, MN, United States \\ ${ }^{2}$ Diakonova University College, Fredensborgveien, Oslo, Norway
}

Received: June 2, 2017

DOI: $10.5430 /$ jnep.v7n11p92
Accepted: June 19, 2017

URL: https://doi.org/10.5430/jnep.v7n11p92

\begin{abstract}
This review analyzes how nurse-led public health interventions promote quality of life (QoL) among older populations. Using Medline and Cinahl databases, authors completed a systematic review of experimental and quasi-experimental studies published between January 2010 and March 2016 that described interventions used by public health nurses to address health needs of older adult populations. Lawton's theoretical QoL concepts and the Public Health Intervention Wheel model, which names interventions at the individual, community and systems levels, were used to interpret results. The 23 studies were widely distributed geographically. Four of Lawton's theoretical QoL domains (Health, Functional Health, Personal Competency, Psychological Well-Being) were addressed in the majority of studies. Although public health nurses used Wheel interventions at all levels of practice, individual level interventions were featured in studies to a greater extent in comparison to community and systems level interventions. Few studies used QoL measures to determine intervention effectiveness. Nurses should consider QoL domains, as they design individual, community, and systems level interventions to improve the health of older adult populations.
\end{abstract}

Key Words: Community, Elder care, Public health nursing, Quality of life, Systematic review

\section{INTRODUCTION}

According to the United Nations (UN) the proportion of people over the age of 60 years is increasing rapidly. It is projected that between 2015 and 2030, the number of older people in the world aged 60 or over is projected to grow from 901 million to 1.4 billion, and by 2050 the global population is projected to more than double its size in 2015 , reaching 2.1 billion globally. ${ }^{[1]}$ In particular, the populations of low income countries will grow significantly with an expected increase to $32 \%$ in $2050 .{ }^{[2]}$ Consequently, many policy makers proclaim that this situation will result in higher expenditures on health and social services. ${ }^{[3]}$ With increasing longevity, aging is rising to the top of the health policy agendas, accompanied with an expanded interest in concepts such as quality of life (QoL) and successful aging. For example, Healthy People 2000, 2010, and 2020 identified QoL improvement as a central public health goal. ${ }^{[4-6]}$ Also, the European Commis$\operatorname{sion}^{[7]}$ has formed the European Innovation Project on Active and Healthy Ageing to improve health and QoL for older people. Such policies suggest that it is in the best interests of societies to promote health and QoL among older adults in order to minimize health care costs and maintain economic productivity.

Although QoL has been defined in many ways, there is a considerable agreement that the concept is multidimensional, and contains physical, psychological, social, environmental, and spiritual dimensions. Broadly speaking one can understand QoL as being about an individual's perception of their

*Correspondence: Kari Glavin; Email: kag@ diakonova.no; Address: Diakonova University College, Fredensborgveien 24Q, N-0177 Oslo, Norway. 
life quality. ${ }^{[8-10]}$ Within the public health context, the term QoL of life corresponds with the 1947 World Health Organization's definition of health, which includes optimal physical, mental, and social functioning. ${ }^{[11]}$ Thus, research on QoL in aging addresses a variety of topics that are related to health, functioning, and longevity. For example, leading areas of research have included the study of the effects of age and aging on survival and mortality; physical functioning and activities of everyday life; cognitive functioning; depression and other psychosocial disorders; falls; injuries; and disease and comorbidities. ${ }^{[12-16]}$ Also, among community-dwelling older adults, a wide variety of factors have been reported to influence QoL including depressive symptoms, physical function, perceived health, environmental conditions, and age, to name a few. ${ }^{[17-23]}$

Demographic aging around the world also means that public health nurses (PHNs) will increasingly find themselves working with older adults. Consequently, understanding what factors may contribute to QoL and changes in QoL has significant implications for the design, implementation, and promotion of public health interventions for older adults, ${ }^{[24]}$ which aim to promote life QoL for all people. ${ }^{[25-27]}$

Consistent with the scope of public health, QoL issues are important for PHNs to consider when planning interventions for older adults. One of the key competencies for PHNs is having the knowledge and skills to incorporate "mental, physical, emotional, social, spiritual, and environmental aspects of health into assessment, planning, implementation, and evaluation" ${ }^{[28]}$ in the provision of nursing care to older adult populations. The holistic approach PHNs bring to their practice will consider all QoL factors that improve health for older adults. This review paper presents an analysis of studies that investigate public health nursing interventions and how these interventions promote QoL of life for older adults.

\section{Methods}

Selected studies described interventions used by PHNs with older adult populations and were published between January 2010 and March 2016 in the English language. Studies were excluded if the intervention did not involve nurses, have a predominant sample of older persons, and were descriptions of study designs without completed results. Also, non-research evidence, such as practice guidelines, case reports, and literature reviews, were excluded. The following definition of intervention was used: "actions taken on the behalf of individuals, families, systems, and communities to improve or protect health status". ${ }^{[29]}$

In consultation with authors, a university librarian completed the searches, using Medline and Cinahl databases.
Search terms for Medline included nursing, nurses, community health nursing, public health nursing, aged, aged over 80 , clinical trials, and nursing interventions. Search terms used in Cinahl were community health nursing, public health nursing, aged, nursing interventions, and experimental studies. These searches resulted in 33 citations in Cinahl and 34 in Medline $(n=67)$. The titles and abstracts of these citations were reviewed independently by three reviewers. During the abstract review process, 24 citations were excluded due to the lack of a precise intervention, a duplicate article, written in a language which was not English, participants not consistent with the age requirement, no comparison to the intervention, or described scenarios and/or hypothetical cases. While screening the remaining full text articles (n $=43$ ), authors excluded an additional 14 studies. In the data extraction process, another 7 articles were excluded based on inclusion criteria. At this point, the authors decided to add the search term "quality of life" to the Medline and Cinahl databases along with the initial search terms. Only one nonduplicate study that met the inclusion criteria resulted from this additional search process. This process resulted in 23 articles for critical review (see Figure 1). All reviewers were in agreement about included studies.

Authors each reviewed a portion of the studies and completed a detailed matrix for each study that included the following components: citation, study location, purpose, framework or theory, setting, method, measures, results, public health interventions, and QoL domains. This matrix was used to critically appraise study quality. Authors used the detailed matrix to create a brief matrix, which specifies authors, study design and intervention, outcomes, and a summary of public health interventions and QoL domains represented in each study.

The Johns Hopkins Nursing Evidence-Based Practice Model provided guidelines for evaluation of study quality. ${ }^{[30]}$ For research evidence (RCTs, quasi-experimental studies, nonexperimental studies), the Johns Hopkins Model specifies criteria for high, good, and low quality research. High quality criteria include: consistent generalizable results, sufficient sample size for study design, adequate control, definitive conclusions, and consistent recommendations. Based on the quality appraisal questions (scored yes, no, not applicable) for research studies identified by Dearholt and Dang, ${ }^{[30]}$ the authors determined that 7 out of the 23 articles met all the criteria for high quality research. Authors identified the following concerns regarding quality: no discussion of instrument reliability or validity ( 9 articles), a large amount of older references or limited references ( 7 articles), small sample size (5 articles), and no discussion of limitations (2 articles). See Table 1 for quality summaries for each study. 


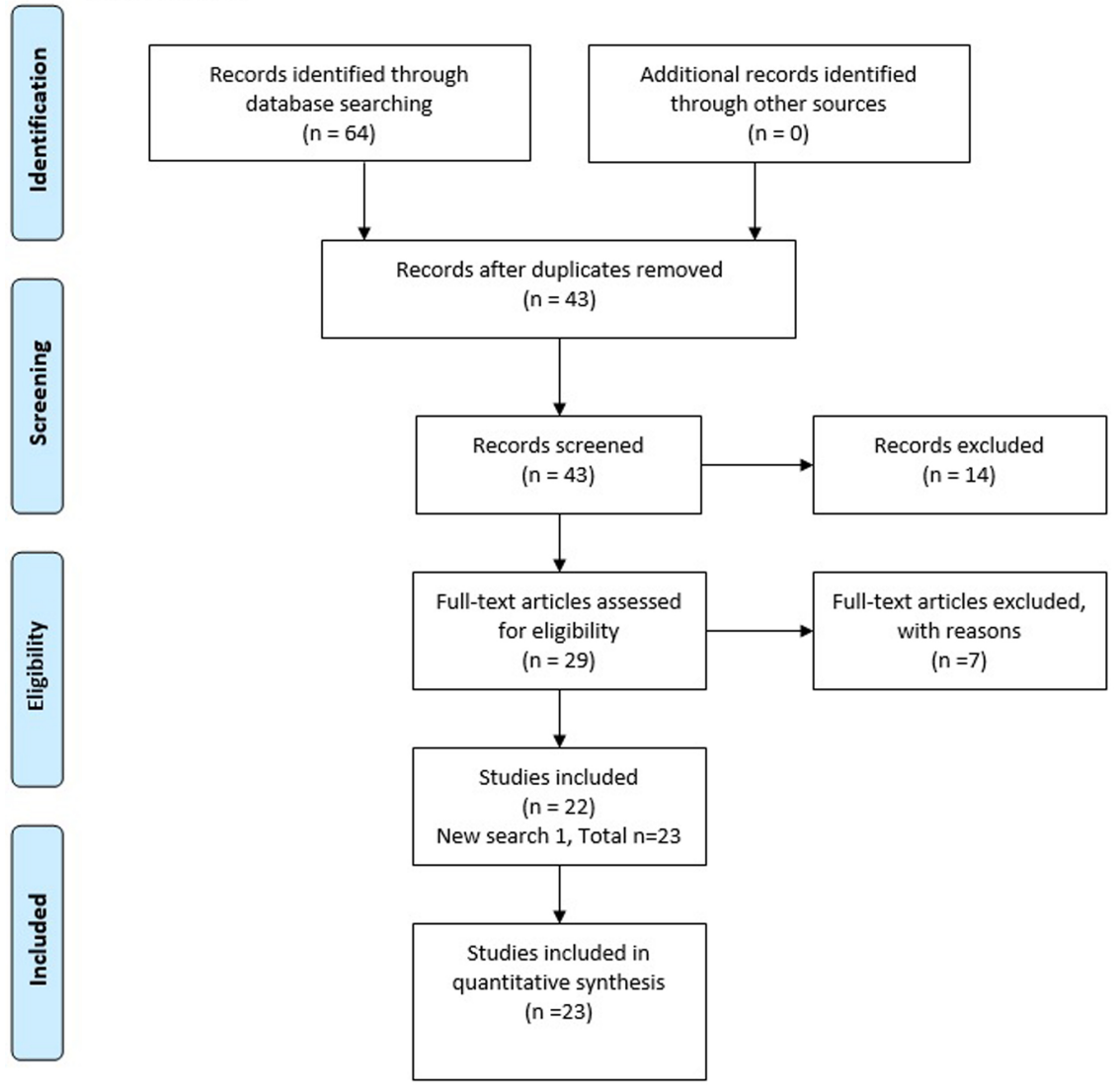

Figure 1. Search process

Although QoL research has increased in methodological rigor, progress has been hindered by the fact that QoL has been used to mean a variety of different things. For example, over a hundred definitions of QoL exist. ${ }^{[31]}$ Moreover, there seems to be no widely accepted theoretical framework for QoL ${ }^{[32]}$ or a general consensus concerning which areas are necessary for any comprehensive definition among adults. Currently, most researchers are in agreement that $\mathrm{QoL}$ among older adults reflects a multidimensional concept, including physical, emotional, social, and spiritual domains; ${ }^{[10,33-37]}$ however, few QoL theories specific to older adults exist. Three decades ago, Lawton ${ }^{[38]}$ described a theory regarding the conceptualization of the "good life" among older adults. QoL became the preferable term in Lawton's later work, replacing the good life. This theory remains popular today, especially because it describes an environmental domain, which is a missing aspect in most QoL definitions. ${ }^{[9,39]}$ Lawton describes Qol as "the multidimensional evaluation, by both intra-personal and socio-economic criteria of the person-environment system of the individual in time past, current, and anticipated" ${ }^{[40]}$ thereby emphasizing QoL as a person-environmental transaction. As reflected in his definition, QoL is also regarded as a multidimensional structure.
Domains which are important to Lawton's conception of QoL include: health, functional health, personal competency, psychological well-being, social behaviour, and environment. See Table 2 for definitions of these domains. The author with research expertise in QoL completed the analysis of relevant QoL domains addressed by outcomes in each study.

For each selected study, two authors, experts in the Public Health Intervention Wheel, identified the nurse-led public health interventions consistent with the Wheel represented in the study designs. ${ }^{[41]}$ The Public Health Intervention Wheel names population-focused interventions that public health professionals and organizations can use to bring about change resulting in improved health status and QoL. Originally developed by PHNs, the Wheel explains what PHNs and other professionals do in their work with individuals, communities, and systems. The Minnesota Department of Health, Section of Public Health Nursing, introduced the Wheel in 1998. PHN consultants conducted an analysis of the literature on population-focused interventions and named interventions based on that analysis. PHNs in practice validated the Intervention Wheel. ${ }^{[41]}$ The Wheel has been disseminated and used as a framework for public health nursing practice across the United States and internationally. 
Table 1. Summary of studies, public health interventions, and quality of life domains

\begin{tabular}{|c|c|c|c|c|c|}
\hline Authors & Study Design & Study Intervention & Results & $\begin{array}{l}\text { Public Health } \\
\text { Interventions }\end{array}$ & $\begin{array}{l}\text { Quality of Life } \\
\text { Domains }\end{array}$ \\
\hline $\begin{array}{l}\text { Austin, Hockey, Williams, \& } \\
\text { Hutchinson }{ }^{[71]} 2013 \\
\text { South Wales } \\
\text { Quality: No discussion of } \\
\text { instrument validity or reliability, no } \\
\text { limitations identified, small sample } \\
\text { size }\end{array}$ & $\begin{array}{l}\text { Quasi-experimental: pre- and } \\
\text { post-measures service } \\
\text { evaluation }(n=25) .14 \mathrm{had} \\
\text { intravenous diuretics; } 11 \text { had } \\
\text { subcutaneous diuretics. }\end{array}$ & $\begin{array}{l}\text { Nurse-led community parental } \\
\text { diuretic service for heart failure } \\
\text { patients. }\end{array}$ & $\begin{array}{l}\text { Decrease in negative } \\
\text { symptoms; high patient } \\
\text { satisfaction ratings }\end{array}$ & $\begin{array}{l}\text { Individual } \\
\text { Case finding } \\
\text { Delegated functions } \\
\text { Systems } \\
\text { Policy development }\end{array}$ & $\begin{array}{l}\text { Health } \\
\text { Psychological } \\
\text { well-being } \\
\text { Personal } \\
\text { competence } \\
\text { Environment }\end{array}$ \\
\hline $\begin{array}{l}\text { Bakas., Austin, Habermann, Jessup } \\
\text { et al. }{ }^{[72]} 2015 \\
\text { United States } \\
\text { Quality: High }\end{array}$ & $\begin{array}{l}\text { RCT, pre- and post-measures } \\
\text { at } 8,12,24 \text {, and } 52 \text { weeks. } \\
\text { Intervention group }(n=123) \text {; } \\
\text { control: information, support, } \\
\text { and referral; comparison group } \\
(n=131) \text {. }\end{array}$ & $\begin{array}{l}\text { Nurse-led Telephone Assessment } \\
\text { and } \\
\text { Skill-Building Kit for caregivers } \\
\text { of stroke patients. }\end{array}$ & $\begin{array}{l}\text { Reduction in depressive } \\
\text { symptoms and improved life } \\
\text { changes for caregivers with } \\
\text { mild to severe depressive } \\
\text { symptoms }\end{array}$ & $\begin{array}{l}\text { Individual } \\
\text { Case finding } \\
\text { Health teaching } \\
\text { Counseling } \\
\text { Consultation }\end{array}$ & $\begin{array}{l}\text { Health } \\
\text { Functional health } \\
\text { Psychological } \\
\text { well-being } \\
\text { Personal } \\
\text { competence }\end{array}$ \\
\hline $\begin{array}{l}\text { Cheng, Cheng, Zhang, Q., Jiang, } \\
\text { Cong, Zang, \& Zhao }{ }^{[73]} 2014 \\
\text { China } \\
\text { Quality: No limitations identified }\end{array}$ & $\begin{array}{l}\text { RCT, patients randomized into } \\
\text { three groups: control group ( } n \\
=30 \text { ), intervention group A } \\
\text { (behavior and chronotherapy } \\
\text { intervention) }(n=30) \text {, and } \\
\text { intervention group B (behavior } \\
\text { intervention) }(n=30)\end{array}$ & $\begin{array}{l}\text { Continuous nursing intervention } \\
\text { (BP monitoring) using } \\
\text { chronotherapeutics (based on } \\
\text { circadian rhythm) for older } \\
\text { hypertensive patients in the } \\
\text { community. }\end{array}$ & $\begin{array}{l}\text { Behavior intervention } \\
\text { improved self-management } \\
\text { ability. No significant BP } \\
\text { change between } 6 \text { and } 12 \\
\text { months after intervention. }\end{array}$ & $\begin{array}{l}\text { Individual } \\
\text { Case finding } \\
\text { Health teaching } \\
\text { Counseling } \\
\text { Consultation }\end{array}$ & $\begin{array}{l}\text { Health } \\
\text { Functional health } \\
\text { Psychological } \\
\text { well-being }\end{array}$ \\
\hline $\begin{array}{l}\text { Cicolini, Simonetti, Comparcini et } \\
\text { al. }{ }^{[74]} 2014 \\
\text { Italy } \\
\text { Quality: High }\end{array}$ & $\begin{array}{l}\text { RCT. Intervention group }(n= \\
\text { 100); control group }(n=98) \text {. } \\
\text { Follow-up at } 1,3 \text {, and } 6 \\
\text { months. }\end{array}$ & $\begin{array}{l}\text { Nurse-led reminder program } \\
\text { through email and phone calls to } \\
\text { improve cardiovascular disease } \\
\text { risk factors among hypertensive } \\
\text { adults. All participants received } \\
\text { usual educational program. }\end{array}$ & $\begin{array}{l}\text { Significant decrease in } \\
\text { prevalence of several } \\
\text { behaviors or risk conditions for } \\
\text { intervention group }\end{array}$ & $\begin{array}{l}\text { Individual } \\
\text { Case management } \\
\text { Health teaching } \\
\text { Counseling } \\
\text { Consultation } \\
\text { Case finding }\end{array}$ & $\begin{array}{l}\text { Health } \\
\text { Functional health } \\
\text { Personal } \\
\text { competence }\end{array}$ \\
\hline $\begin{array}{l}\text { Duffy, Hoskins, \& Dudley- } \\
\text { Brown }{ }^{[75]} 2010 \\
\text { United States } \\
\text { Quality: Small sample size }\end{array}$ & $\begin{array}{l}\text { RCT. Intervention group } \\
(n=15) \text {, control group received } \\
\text { usual home visits ( } \mathrm{n}=17)\end{array}$ & $\begin{array}{l}\text { Telephone and home visits } \\
\text { (structured telephone script } \\
\text {-symptoms, education, emotional } \\
\text { support) delivered by home health } \\
\text { nurses over } 6 \text { weeks. }\end{array}$ & $\begin{array}{l}\text { QoL }-8.6 \% \text { improvement for } \\
\text { intervention group, not } \\
\text { significant. Intervention group } \\
\text { more satisfied with home } \\
\text { health services at end of } 60 \\
\text { days. }\end{array}$ & $\begin{array}{l}\text { Individual } \\
\text { Health teaching } \\
\text { Counseling } \\
\text { Case finding }\end{array}$ & $\begin{array}{l}\text { Health } \\
\text { Biologic health } \\
\text { Functional health } \\
\text { Psychological } \\
\text { well-being } \\
\text { Personal } \\
\text { competence } \\
\text { Environment }\end{array}$ \\
\hline $\begin{array}{l}\text { Friedman, Li, Liebel, \& } \\
\text { Powers }{ }^{[76]} 2014 \\
\text { United States } \\
\text { Quality: References include many } \\
\text { older studies }\end{array}$ & $\begin{array}{l}\text { RCT, secondary analysis } \\
\text { comparing two arms of the } \\
\text { Medicare PCDC } \\
\text { Demonstration at } 22 \text { months } \\
\text { after study entry. Home } \\
\text { visiting nurse (HVN) } \\
\text { intervention group }(n=237) \text {; } \\
\text { control group - usual care }(n= \\
262)\end{array}$ & $\begin{array}{l}\text { Nurse home visit intervention } \\
\text { focused on empowering patients } \\
\text { and education about the use of } \\
\text { behavioral change models to } \\
\text { facilitate chronic disease } \\
\text { management. }\end{array}$ & $\begin{array}{l}\text { HVN intervention group had } \\
\text { less difficulty bathing at } 22 \\
\text { months and less dependence in } \\
\text { walking and transferring. No } \\
\text { effects for difficulty } \\
\text { performing the other IADLs or } \\
\text { for lower levels of dependence } \\
\text { for any ADLs. }\end{array}$ & $\begin{array}{l}\text { Individual } \\
\text { Case management } \\
\text { Case finding } \\
\text { Health teaching } \\
\text { Counseling }\end{array}$ & $\begin{array}{l}\text { Health } \\
\text { Biological health } \\
\text { Functional health } \\
\text { Psychological } \\
\text { well-being } \\
\text { Environment }\end{array}$ \\
\hline $\begin{array}{l}\text { Hunger, Kirchberger. Holle et al. } \\
{ }^{[78]}{ }_{2015} \\
\text { Germany } \\
\text { Quality: No discussion of } \\
\text { instrument validity }\end{array}$ & $\begin{array}{l}\text { RCT, two-armed parallel } \\
\text { group trial. Intervention group } \\
(n=116) \text {; control group usual } \\
\text { care }(n=132) \text {. One year } \\
\text { follow-up. }\end{array}$ & $\begin{array}{l}\text { Nurse-led program to reduce } \\
\text { atherogenic risk factors, physical } \\
\text { functioning and mental health. } \\
\text { Intervention: home visits and } \\
\text { telephone calls. }\end{array}$ & $\begin{array}{l}\text { Intervention group had } \\
\text { significantly better } \\
\text { low-density lipoprotein } \\
\text { cholesterol levels, hand grip } \\
\text { strength, and nutrition risk } \\
\text { scores. Also had better mean } \\
\text { scores on self-reported mental } \\
\text { health, disability, and ADLs. }\end{array}$ & $\begin{array}{l}\text { Individual } \\
\text { Case management } \\
\text { Case finding } \\
\text { Health teaching } \\
\text { Counseling } \\
\text { Consultation } \\
\text { Referral and } \\
\text { follow-up }\end{array}$ & $\begin{array}{l}\text { Health } \\
\text { Functional health } \\
\text { Psychological well- } \\
\text { being } \\
\text { Personal } \\
\text { competence }\end{array}$ \\
\hline $\begin{array}{l}\text { Imhof, Naef, Wallhagen, Schwartz, } \\
\text { \& Mahrer-Imhof }{ }^{[79]} 2012 \\
\text { Switzerland } \\
\text { Quality: No discussion of } \\
\text { instrument reliability and validity } \\
\text { but cited references }\end{array}$ & $\begin{array}{l}\text { RCT, longitudinal. } \\
\text { Intervention group - home } \\
\text { visits after } 4,12,24 \text {, and } 36 \\
\text { weeks; } 3 \text { telephone calls after } \\
8,18 \text { and } 30 \text { weeks. Control } \\
\text { group }(n=207) ; \text { standard care } \\
(n=206) .\end{array}$ & $\begin{array}{l}\text { Advanced practice nurse (APN) } \\
\text { in-home health consultation } \\
\text { program (HCP). } 4 \text { in-home visits } \\
\text { and } 3 \text { phone calls from APNs. }\end{array}$ & $\begin{array}{l}\text { No significant difference on } \\
\text { QoL measure. Benefits over } 9 \\
\text { months for self-reported acute } \\
\text { events, falls, consequences of } \\
\text { falls, and hospitalizations for } \\
\text { intervention group. }\end{array}$ & $\begin{array}{l}\text { Individual } \\
\text { Health teaching } \\
\text { Counseling } \\
\text { Consultation } \\
\text { Case management }\end{array}$ & $\begin{array}{l}\text { Health } \\
\text { Psychological well- } \\
\text { being } \\
\text { Personal } \\
\text { competence } \\
\text { Social functioning } \\
\text { Environment }\end{array}$ \\
\hline $\begin{array}{l}\text { Jansen, van Hout, Nijpels et al. }{ }^{[80]} \\
2011 \\
\text { Netherlands } \\
\text { Quality: No discussion of } \\
\text { instrument reliability or validity but } \\
\text { cited references }\end{array}$ & $\begin{array}{l}\text { RCT, case management by } \\
\text { district nurses }(n=54) \text { or usual } \\
\text { care }(n=45) \text {. Patient/ } \\
\text { caregiver dyads. }\end{array}$ & $\begin{array}{l}\text { Nurses provided assessment, } \\
\text { advice and information, planning, } \\
\text { coordinating, organizing } \\
\text { collaboration, and monitoring of } \\
\text { care. }\end{array}$ & $\begin{array}{l}\text { No benefits of case } \\
\text { management for older adults } \\
\text { with dementia symptoms and } \\
\text { their primary caregivers over } \\
\text { usual care. } \\
\text { Informal caregivers satisfied } \\
\text { with quality of case } \\
\text { management. }\end{array}$ & $\begin{array}{l}\text { Individual, } \\
\text { Community } \\
\text { Case management } \\
\text { Screening } \\
\text { Collaboration } \\
\text { Health teaching, } \\
\text { Referral and } \\
\text { follow-up } \\
\text { Individual } \\
\text { Case finding }\end{array}$ & $\begin{array}{l}\text { Health } \\
\text { Psychological } \\
\text { well-being } \\
\text { Social behavior } \\
\text { Personal } \\
\text { competence }\end{array}$ \\
\hline $\begin{array}{l}\text { Kerr, Whyte, Watson, Tolson, \& } \\
\text { McFadyen }{ }^{[81]} 2011 \\
\text { Scotland } \\
\text { Quality: References include many } \\
\text { older studies }\end{array}$ & $\begin{array}{l}\text { RCT, two group parallel } \\
\text { design; community nurses and } \\
\text { allied health professionals; } \\
\text { intervention group }(n=27 \text {, } \\
\text { control group }(n=25) \text {. } \\
\text { Semi-structured qualitative } \\
\text { interviews }(n=8)\end{array}$ & $\begin{array}{l}\text { Intervention - a one-day brief } \\
\text { intervention smoking cessation } \\
\text { training. }\end{array}$ & $\begin{array}{l}\text { Statistically significant } \\
\text { improvement in knowledge } \\
\text { and attitudes of the } \\
\text { intervention group. Qualitative } \\
\text { findings demonstrated how } \\
\text { training impacted positively on } \\
\text { practice. }\end{array}$ & $\begin{array}{l}\text { Systems } \\
\text { Health teaching } \\
\text { Counseling } \\
\text { Consultation }\end{array}$ & $\begin{array}{l}\text { Health } \\
\text { Functional health } \\
\text { Personal } \\
\text { competency }\end{array}$ \\
\hline $\begin{array}{l}\text { Kim, Ko, } \\
\text { Lee, \& Kim }{ }^{[82]} 2014 \\
\text { South Korea } \\
\text { Quality: Small sample size }\end{array}$ & $\begin{array}{l}\text { Quasi-Experimental, pretest - } \\
\text { posttest design. Low income } \\
\text { adults with hypertension ( } n= \\
22 \text { ) in a public health center }\end{array}$ & $\begin{array}{l}\text { Intervention: case management } \\
\text { program delivered by visiting } \\
\text { nurses (home visits and telephone } \\
\text { contacts). }\end{array}$ & $\begin{array}{l}\text { Significant changes in mean } \\
\text { scores for lifestyle, physical } \\
\text { activity, diet, and medication. } \\
\text { Increase in hypertension } \\
\text { knowledge. }\end{array}$ & $\begin{array}{l}\text { Individual } \\
\text { Case management } \\
\text { Health teaching } \\
\text { Counseling } \\
\end{array}$ & $\begin{array}{l}\text { Health } \\
\text { Functional health }\end{array}$ \\
\hline
\end{tabular}

(Table 1 continued on page 96) 
Table 1. (continued)

\begin{tabular}{|c|c|c|c|c|c|}
\hline Authors & Study Design & Study Intervention & Results & $\begin{array}{l}\text { Public Health } \\
\text { Interventions }\end{array}$ & $\begin{array}{l}\text { Quality of Life } \\
\text { Domains }\end{array}$ \\
\hline $\begin{array}{l}\text { Li, Liebel, \& Friedman }{ }^{[83]} 2013 \\
\text { United States } \\
\text { Quality: No discussion of } \\
\text { instrument reliability and validity, } \\
\text { mixture of old and new references }\end{array}$ & $\begin{array}{l}\text { RCT, secondary analysis } \\
\text { comparing two arms of the } \\
\text { Medicare PCDC Demonstration } \\
\text { at } 22 \text { months after study entry. } \\
\text { Home visiting nurse (HVN) } \\
\text { intervention group }(n=237) ; \\
\text { control group - usual care }(n= \\
\text { 262) }\end{array}$ & $\begin{array}{l}\text { Intervention: monthly home } \\
\text { visits by trained nursing staff. }\end{array}$ & $\begin{array}{l}\text { HVN group had less difficulty/ } \\
\text { dependence for meal preparation, } \\
\text { telephone use, shopping and } \\
\text { ordinary housework, and more } \\
\text { difficulty/ dependence for } \\
\text { medication management. HVN } \\
\text { intervention had effects on } \\
\text { several but not all IADLs. }\end{array}$ & $\begin{array}{l}\text { Individual } \\
\text { Case management }\end{array}$ & $\begin{array}{l}\text { Health } \\
\text { Functional health } \\
\text { Psychological } \\
\text { well-being } \\
\text { Environment }\end{array}$ \\
\hline $\begin{array}{l}\text { Marquez-Serrano, Gonzalez-Juarez } \\
\text { Castillo-Castillo, } \\
\text { Gonzalez-Gonzalez, \& Idravo }{ }^{[84]} \\
2012 \\
\text { Mexico } \\
\text { Quality: Limited references, no } \\
\text { discussion of instrument reliability } \\
\text { and validity, small sample size }\end{array}$ & $\begin{array}{l}\text { Quasi-experimental, before and } \\
\text { after intervention design. } \\
\text { Egocentric social network } \\
\text { analysis to explore transition of } \\
\text { knowledge in elders' network. } \\
\text { Snowball sampling of elders over } \\
60 \text { years }(n=10) \text {. Social network } \\
(n=94)\end{array}$ & $\begin{array}{l}\text { Nursing intervention: seven } \\
\text { educational sessions on acute } \\
\text { respiratory infections to } \\
\text { improve self-care. }\end{array}$ & $\begin{array}{l}\text { Knowledge of the correct use of } \\
\text { antibiotics increased. Incidence of } \\
\text { respiratory infections in social } \\
\text { network did not decrease. }\end{array}$ & $\begin{array}{l}\text { Individual } \\
\text { Health teaching } \\
\text { Counseling } \\
\text { Case finding }\end{array}$ & $\begin{array}{l}\text { Health } \\
\text { Psychological } \\
\text { well-being } \\
\text { Personal } \\
\text { competence } \\
\text { Social functioning }\end{array}$ \\
\hline $\begin{array}{l}\text { Meethien, Pothiban, Ostwald, } \\
\text { Sucamvang, \& Panuthai }{ }^{\left[{ }^{[5]}\right.} 2011 \\
\text { Thailand } \\
\text { Quality: Many older references }\end{array}$ & $\begin{array}{l}\text { RCT, experimental group elders } \\
\text { and family members ( } n=43 \\
\text { pairs); control group ( } n=43 \\
\text { pairs) had usual care. }\end{array}$ & $\begin{array}{l}\text { Intervention: Nurse-led } \\
\text { nutritional education program } \\
\text { to increase nutritional } \\
\text { knowledge/skills, perceived } \\
\text { self-efficacy, and benefits for } \\
\text { healthy eating. }\end{array}$ & $\begin{array}{l}\text { Experimental group had } \\
\text { significantly higher scores on } \\
\text { overall healthy eating and healthy } \\
\text { eating sub-dimensions. Also had } \\
\text { higher healthy eating scores, one } \\
\text { week and } 12 \text { weeks after } \\
\text { completing the education } \\
\text { program. }\end{array}$ & $\begin{array}{l}\text { Iindividual } \\
\text { Case management } \\
\text { Health teaching } \\
\text { Counseling } \\
\text { Consultation } \\
\text { Case finding }\end{array}$ & $\begin{array}{l}\text { Health } \\
\text { Functional health } \\
\text { Personal } \\
\text { competency }\end{array}$ \\
\hline $\begin{array}{l}\text { Rodríguez-Cerrillo, Poza-Montoro, } \\
\text { Fernandez-Diaz, Matesanz-David, \& } \\
\text { Romero }{ }^{[86]} 2013 \\
\text { Spain } \\
\text { Quality: Limited and older } \\
\text { references, small sample size, no } \\
\text { discussion of instrument reliability } \\
\text { or validity }\end{array}$ & $\begin{array}{l}\text { Quasi-experimental prospective } \\
\text { study, including patients over } 70 \\
\text { years with uncomplicated } \\
\text { diverticulitis admitted to Hospital } \\
\text { at Home Unit }(n=34) \text { and to } \\
\text { Conventional Hospitalization ( } n= \\
\text { 18) from the Emergency } \\
\text { Department. }\end{array}$ & $\begin{array}{l}\text { Nurse visits of elderly patients } \\
\text { with uncomplicated } \\
\text { diverticulitis treated at home } \\
\text { versus traditional } \\
\text { hospitalization }\end{array}$ & $\begin{array}{l}\text { No patients treated at home were } \\
\text { transferred to hospital. Home } \\
\text { treatment was associated with a } \\
\text { cost reduction of } 1368 \text { euros per } \\
\text { patient. }\end{array}$ & $\begin{array}{l}\text { Individual } \\
\text { Case management } \\
\text { Health teaching } \\
\text { Delegation }\end{array}$ & $\begin{array}{l}\text { Health } \\
\text { Functional health } \\
\text { Personal } \\
\text { competency } \\
\text { Environmental }\end{array}$ \\
\hline $\begin{array}{l}\text { Rosted, Poulsen Hendriksen, } \\
\text { Petersen, \& Wagner }{ }^{[87]} 2013 \\
\text { Denmark } \\
\text { Quality: High }\end{array}$ & $\begin{array}{l}\text { Prospective RCT of patients } 70 \\
\text { and over admitted to an ER. } \\
\text { Intervention group }(n=141) \text {; } \\
\text { control group ( }(n=130) \text {. } \\
\text { Follow-up at } 30 \text { and } 180 \text { days } \\
\text { following discharge. }\end{array}$ & $\begin{array}{l}\text { Two-stage nursing assessment } \\
\text { and problem-solving } \\
\text { intervention to make a plan to } \\
\text { resolve problems. }\end{array}$ & $\begin{array}{l}\text { At } 180 \text { days, } 26 \% \text { from } \\
\text { intervention group readmitted, vs } \\
38 \% \text { of control group. No } \\
\text { significant differences in physical } \\
\text { functioning between groups at } \\
\text { baseline and follow-up. } \\
\text { Intervention group- less likely to } \\
\text { be depressed at } 180 \text { days. }\end{array}$ & $\begin{array}{l}\text { Individual } \\
\text { Health teaching } \\
\text { Counseling } \\
\text { Case finding } \\
\text { Referral and } \\
\text { Follow-up } \\
\text { Consultation } \\
\text { Case Management }\end{array}$ & $\begin{array}{l}\text { Health } \\
\text { Functional health } \\
\text { Social behavior } \\
\text { Psychological } \\
\text { well-being } \\
\text { Personal } \\
\text { competence } \\
\text { Environment }\end{array}$ \\
\hline $\begin{array}{l}\text { Rytter, Jakosen, \& Ronholt et al. }{ }^{[88]} \\
2010 \\
\text { Denmark } \\
\text { Quality: Many older references }\end{array}$ & $\begin{array}{l}\text { RCT, intervention group ( } n= \\
\text { 148). Control group }(n=145) \\
\text { received usual care. }\end{array}$ & $\begin{array}{l}\text { Interdisciplinary care given by } \\
\text { GPs and district nurses. } \\
\text { Structured home visit by the } \\
\text { GP and the district nurse one } \\
\text { week after discharge followed } \\
\text { by two contacts after three and } \\
\text { eight weeks. }\end{array}$ & $\begin{array}{l}\text { Significant readmission after } 26 \\
\text { weeks - } 52 \% \text { patients in control } \\
\text { group, } 40 \% \text { in intervention group. } \\
\text { Cost-neutral; tendency to reduce } \\
\text { costs. }\end{array}$ & $\begin{array}{l}\text { Community, } \\
\text { Systems } \\
\text { Collaboration } \\
\text { Systems } \\
\text { Surveillance } \\
\text { Case management }\end{array}$ & $\begin{array}{l}\text { Health } \\
\text { Functional health }\end{array}$ \\
\hline $\begin{array}{l}\text { Shearer, Fleury, \& Belyea }{ }^{[89]} 2010 \\
\text { United States } \\
\text { Quality: High }\end{array}$ & $\begin{array}{l}\text { RCT; HEI intervention }(n=20) \text {, } \\
\text { comparison group }(n=22) \\
\text { received a newsletter focused on } \\
\text { health and safety issues relevant } \\
\text { to older adults. }\end{array}$ & $\begin{array}{l}\text { Health Empowerment } \\
\text { Intervention (HEI). Weekly } \\
\text { nurse visits for } 6 \text { weeks to } \\
\text { facilitate participant } \\
\text { engagement in recognizing } \\
\text { personal resources, social } \\
\text { contextual resources, and } \\
\text { desired health goals. }\end{array}$ & $\begin{array}{l}\text { Older, frailer male participants } \\
\text { with more chronic conditions and } \\
\text { less money seemed to benefit the } \\
\text { most from the intervention. }\end{array}$ & $\begin{array}{l}\text { Individual } \\
\text { Counseling } \\
\text { Referral and } \\
\text { follow-up }\end{array}$ & $\begin{array}{l}\text { Health } \\
\text { Psychological } \\
\text { well-being } \\
\text { Social behavior } \\
\text { Personal } \\
\text { competence } \\
\text { Environment }\end{array}$ \\
\hline $\begin{array}{l}\text { Søgaard, Sørensen, Waldorff,, } \\
\text { Eckermann, Buss, \& Waldemar }{ }^{[90]} \\
2014 \\
\text { Denmark } \\
\text { Quality: High }\end{array}$ & $\begin{array}{l}\text { RCT; random assignment to } \\
\text { intervention group }(n=163) \text { and } \\
\text { control group }(n=167) \text {. }\end{array}$ & $\begin{array}{l}\text { Intervention: Counseling } \\
\text { sessions provided by nurse for } \\
\text { patients with Alzheimer's } \\
\text { disease and their primary } \\
\text { caregiver. }\end{array}$ & $\begin{array}{l}\text { No difference in resource use or } \\
\text { costs between the groups. Trends } \\
\text { were a lower use of formal health } \\
\text { care and nursing homes but a } \\
\text { substantially higher use of } \\
\text { informal care for intervention } \\
\text { group. }\end{array}$ & $\begin{array}{l}\text { Individual, } \\
\text { Systems } \\
\text { Health teaching } \\
\text { Counseling }\end{array}$ & $\begin{array}{l}\text { Health } \\
\text { Psychological } \\
\text { well-being } \\
\text { Social behavior }\end{array}$ \\
\hline $\begin{array}{l}\text { Wang, Chen, Liao, \& Hsiao }{ }^{[91]} 2013 \\
\text { Tawain } \\
\text { Quality: Many older references }\end{array}$ & $\begin{array}{l}\text { RCT, stroke patients. } \\
\text { Intervention group }(n=65) \text {, } \\
\text { normal care group }(n=62) \text {. } \\
\text { Groups were assessed at baseline, } \\
\text { after intervention, and at 6-month } \\
\text { follow-up. }\end{array}$ & $\begin{array}{l}\text { Intervention: } \\
\text { community-based stroke } \\
\text { nursing education and } \\
\text { rehabilitation } \\
\text { Program - stroke patients } \\
\text { received three 2-hour stroke } \\
\text { interventions per week for } 8 \\
\text { weeks. }\end{array}$ & $\begin{array}{l}\text { Significant improvement in } \\
\text { knowledge scores including } \\
\text { warning signs and medical } \\
\text { Treatment, risk factors of stroke, } \\
\text { and dietary factors in the } \\
\text { intervention group. }\end{array}$ & $\begin{array}{l}\text { Individual, } \\
\text { Community } \\
\text { Health teaching } \\
\text { Counseling } \\
\text { Outreach } \\
\text { Community } \\
\text { Collaboration }\end{array}$ & $\begin{array}{l}\text { Health } \\
\text { Functional health } \\
\text { Social behavior } \\
\text { Personal } \\
\text { competence }\end{array}$ \\
\hline $\begin{array}{l}\text { Wong, Chow, Chan, \& Tam }{ }^{[92]} \\
2014 \\
\text { China } \\
\text { Quality: High }\end{array}$ & $\begin{array}{l}\text { RCT with } 3 \text { groups: control }(n= \\
210) \text {, home visits with calls }(n= \\
196) \text {, and calls only }(n=204) .\end{array}$ & $\begin{array}{l}\text { Intervention: Transitional care } \\
\text { program for group of } \\
\text { discharged patients with } \\
\text { chronic diseases. Nurses } \\
\text { provided home visits with calls } \\
\text { and calls only. }\end{array}$ & $\begin{array}{l}\text { Home visit group had a lower } \\
\text { readmission rate. No significant } \\
\text { difference for call group. } \\
\text { Significant improvement in } \\
\text { quality of life, self-efficacy, and } \\
\text { satisfaction for intervention } \\
\text { groups. }\end{array}$ & $\begin{array}{l}\text { Individual } \\
\text { Health teaching } \\
\text { Collaboration } \\
\text { Referral and } \\
\text { follow-up }\end{array}$ & $\begin{array}{l}\text { Health } \\
\text { Functional health } \\
\text { Social functioning } \\
\text { Psychological } \\
\text { well-being } \\
\text { Personal } \\
\text { competency } \\
\text { Environment }\end{array}$ \\
\hline $\begin{array}{l}\text { Young, Trivedi, \& Dickinson }{ }^{[93]} \\
2011 \\
\text { International } \\
\text { Quality: High }\end{array}$ & $\begin{array}{l}\text { Systematic review of RCTs. } \\
\text { Data sources: PUBMED, } \\
\text { CINAHL, PSYCINFO, the } \\
\text { Cochrane Central Register of } \\
\text { Controlled Trials and the National } \\
\text { Research Register. }\end{array}$ & $\begin{array}{l}\text { Evaluated effectiveness of } \\
\text { nutritional education or advice } \\
\text { delivered by health } \\
\text { professionals, including } 10 \\
\text { nurses. }\end{array}$ & $\begin{array}{l}23 \text { studies; nutritional education } \\
\text { or advice can be used to positively } \\
\text { influence diet and improve } \\
\text { physical function. Interventions, } \\
\text { with nutritional education can } \\
\text { also reduce depression. }\end{array}$ & $\begin{array}{l}\text { Iindividual } \\
\text { Case management } \\
\text { Health teaching } \\
\text { Counseling } \\
\text { Consultation } \\
\text { Referral and } \\
\text { follow-up }\end{array}$ & $\begin{array}{l}\text { Health } \\
\text { Functional health } \\
\text { Psychological } \\
\text { well-being } \\
\text { Personal } \\
\text { competence } \\
\text { Environment }\end{array}$ \\
\hline
\end{tabular}


Table 2. Frequencies in Lawton's quality of life domains

\begin{tabular}{l}
\hline Domain \\
Health (all studies) \\
Includes indicators focused on cell, organ, system, and body. Objective \\
indicators such as observable symptoms and measurable physiologica \\
function. No absolute criterion for health. \\
Functional Health (20) \\
Includes indicators such as physical activities of daily living (ADL), \\
instrumental ADL, financial management, and paid employment.
\end{tabular}

Personal Competency (18)

Includes indicators of behavioral competence and social-normative evaluations of functioning in health, cognitive, time use, and social dimensions.

Psychological Well-Being (16)

Includes indicators such as mental health, sensory reception, perception, memory, classical conditioning, operant conditioning symbolic thinking, problem solving, creative innovation, overall satisfaction, positive/negative emotions.

\section{Environment (10)}

Includes indicators such as air and water quality, physical structure of the environment, home, neighborhood, interpersonal relationships, physical accessibility, and factors impacting quality of life.

\section{Social Behavior (9)}

Includes indicators such as sensory contact, casual contact, intimacy, nurturance, creative leadership, love, parenthood, altruistic behavior.

\section{Focus in Studies}

Biological health $(n=16)$

Physical health $(n=3)$

Lifestyle $(n=3)$

Health status $(n=2)$

Pain, hearing, vision $(n=1)$

Symptoms $(n=1)$

Self-maintenance skills $(n=16)$

Activities of daily living $(n=14)$

Physical $(n=8)$

Instrumental activities of daily living $(n=4)$

Mobility $(n=3)$

Money management $(n=2)$

Self-efficacy $(n=4)$

Educational knowledge $(n=3)$

Overall satisfaction $(n=2)$

Subjective evaluation $(n=2)$

Subjective well-being $(n=2)$

Coping $(n=2)$

Self-determination/goals $(n=1)$

Behavioral affect $(n=1)$

Freedom/independence $(n=1$

Behavioral change $(n=1)$

Caregiver competency $(n=1)$

Cognitive judgements $(n=18)$

Mental health $(n=9)$

Self -perception $(n=6)$

Negative emotion $(n=2)$

Concerns $(n=1)$

Emotions $(n=1)$

Positive emotions $(n=1)$

Stress $(n=1)$

Physical environment $(n=3)$

Health service utilization $(n=2)$

Housing $(n=1)$

Physical accessibility $(n=1)$

Mobility ( $n=1)$

Living arrangements $(n=1)$

Community service $(n=1)$

Service quality/cost $(n=1)$

Home environment $(n=1)$

Social interaction $(n=5)$

Social network $(n=2)$

Family $(n=2)$

Sensory $(n=1)$

Friends $(n=1)$

Colleagues $(n=1)$

Belonging $(n=1)$

Nurturance $(n=1)$

Social service $(n=1)$

Emotional support $(n=1)$

Social network $(n=1)$

Note. Source: Lawton, M.P., 1991. A multidimensional view of quality of life in frail elders. J.E. Birren et al. (Eds.), The Concept and Measurement of Quality of Life in the Frail Elderly. Academic Press, San Diego, pp. 3-25. 
The Wheel includes 17 interventions and three levels of practice $^{[29]}$ (see Figure 2). The interventions are organized into five colored wedges, grouping interventions that often occur together. Table 3 identifies the interventions and definitions. At the individual level, interventions focus on changing the "knowledge, attitudes, beliefs, practices, and behaviors of individuals". ${ }^{[41]}$ At the community level, interventions are aimed at changing "community norms, community attitudes, community awareness, community practices, and community behaviors". ${ }^{[41]}$ When practitioners intervene at the systems level, the goal is to change "organizations, policies, laws, and power structures. The focus is on the systems that impact health, not directly on individuals and communities". ${ }^{[41]}$ All interventions are aimed at improving population health. Additional assumptions of the framework include: 1) interventions are based on community assessment, 2) they take into account broad determinants of health, and 3) they emphasize health promotion and disease prevention.

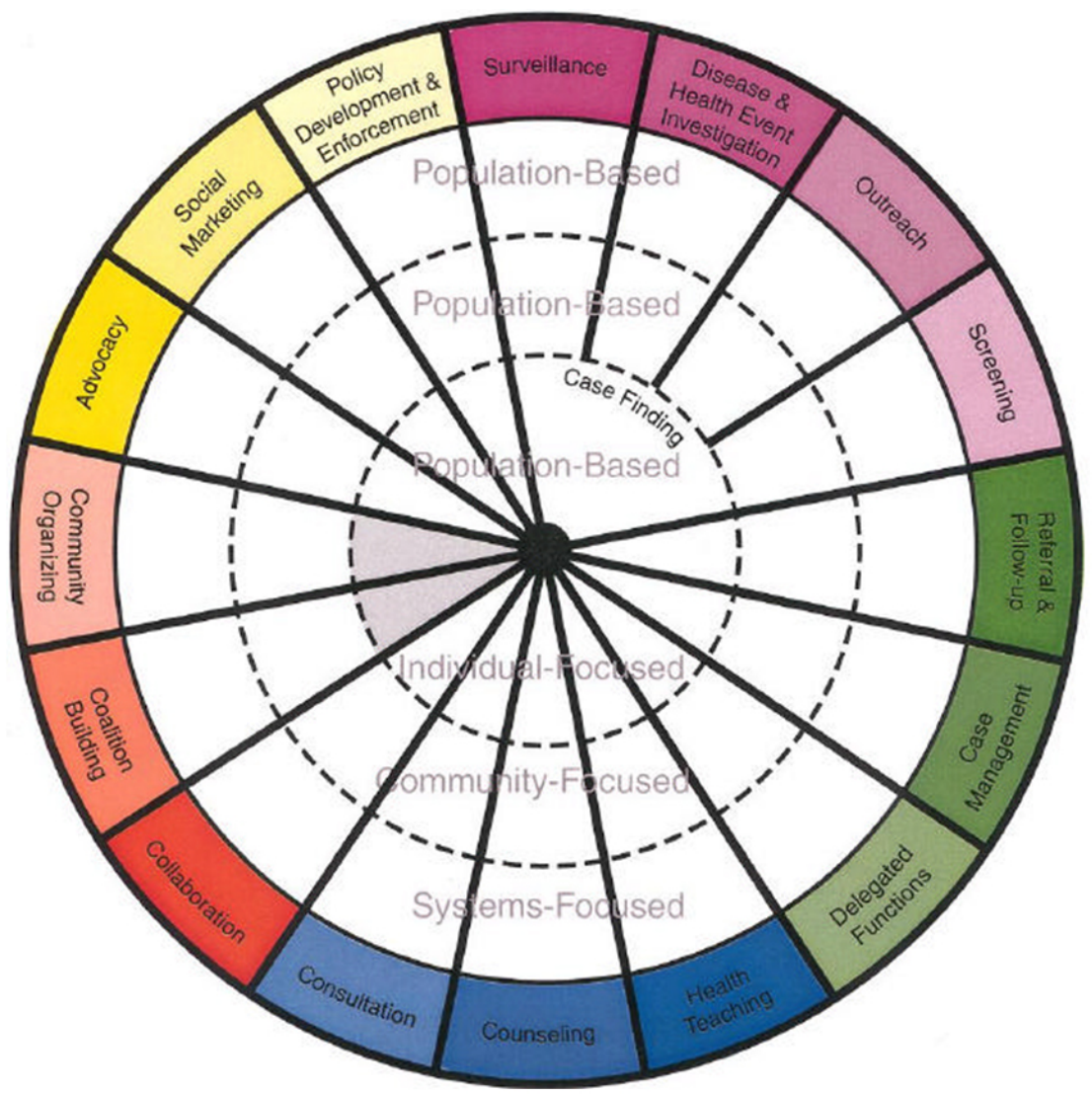

Figure 2. Public health intervention wheel

Although most interventions are used at all three levels of practice, there is some variability. Case-finding is the individual level for all four red wedge interventions (surveillance, disease and health event investigation, and outreach). In the orange wedge, two interventions (coalition building and community organizing) are practiced only at the community and systems levels.

\section{RESUlts}

Of the 23 review articles, there were 17 randomized controlled trials (RCTs), 4 quasi-experimental studies, one systematic review of RCTs, and a mixed-method study with RCT and qualitative arms. The 23 review articles represented a wide geographic region: nine European studies, five from Asia, five from North America, three from Scandinavia, and one from Central America. There were no studies from Africa or Oceania. There was little consistency in use of theory or frameworks across the studies, consequently, choice of measurements used in the intervention were not theory-driven. Eleven studies made no connection to theory. No one theoretical perspective predominated for the remaining 12 studies. Examples of theories used include health promotion, caring models, and self-efficacy. We also have data on what type of instruments were used, which emphasize health, functional status, psychological well-being, and personal competence but have less frequency of socialenvironmental measurements. 
Table 3. Public health interventions in nurse-led programs

\begin{tabular}{|c|c|}
\hline Intervention Definition & Level (Frequenc \\
\hline RFD & \\
\hline $\begin{array}{l}\text { Surveillance: Describes and monitors health events through ongoing and systematic collection, analysis, and } \\
\text { interpretation of health data for the purpose of planning, implementing, and evaluating public health } \\
\text { interventions. }\end{array}$ & $\begin{array}{l}\text { Community (1) } \\
\text { Systems (1) }\end{array}$ \\
\hline
\end{tabular}

\section{Disease and other health event investigation:}

Systematically gathers and analyzes data regarding threats to the health of populations, ascertains the source of 0 the threat, identifies cases and others at risk, and determines control measures.

Outreach: Locates populations-of-interest or populations-at-risk and provides information about the nature of the concern, what can be done about it, and how services can be obtained.

Community (1)

Screening: Identifies individuals with unrecognized health risk factors or asymptomatic disease conditions in populations.

Case-finding: Locates individuals and families with identified risk factors and connects them with resources (individual level intervention for surveillance, disease and health event investigation, outreach, and screening).

\section{GREEN WEDGE}

Referral and follow-up: Assists individuals, families, groups, organizations, and/or communities to identify and access necessary resources in to prevent or resolve problems or concerns.

Case management: Optimizes self-care capabilities of individuals and families and the capacity of systems and communities to coordinate and provide services.

Community (1)

Individual (11)

Delegated functions: Direct care tasks a registered professional nurse carries out under the authority of a

health care practitioner as allowed by law. Delegated functions also include any direct care tasks a registered Individual (2) professional nurse entrusts to other appropriate personnel to perform.

\section{BLUE WEDGE}

Health teaching: Communicates facts, ideas and skills that change knowledge, attitudes, values, beliefs, behaviors, and practices of individuals, families, systems, and/or communities.

Counseling: Establishes an interpersonal relationship with a community, a system, family or individual intended to increase or enhance their capacity for self-care and coping. Counseling engages the community, a system, family or individual at an emotional level.

Individual (5) Community (1) Individual (10) Community (1) Systems (1) Consultation: Seeks information and generates optional solutions to perceived problems or issues through Individual (17) Community (2) Systems (2) Individual (15) Community (1) Systems (2) interactive problem solving with a community, system, family or individual. The community, system, family Systems (1) or individual selects and acts on the option best meeting the circumstances.

\section{ORANGE WEDGE}

Collaboration: Commits two or more persons or organizations to achieve a common goal through enhancing the capacity of one or more of the members to promote and protect health.

Individual (2)

Community (3) Systems (2)

Coalition building: Promotes and develops alliances among organizations or constituencies for a common purpose. It builds linkages, solves problems, and/or enhances local leadership to address health concerns.

Community organizing: Helps community groups to identify common problems or goals, mobilize resources, and develop and implement strategies for reaching the goals they collectively have set.

\section{YELLOW WEDGE}

Advocacy: Pleads someone's cause or act on someone's behalf, with a focus on developing the community, system, individual or family's capacity to plead their own cause or act on their own behalf.

Social marketing: Utilizes commercial marketing principles and technologies for programs designed to influence the knowledge, attitudes, values, beliefs, behaviors, and practices of the population-of- interest.

Policy development: Places health issues on decision-makers' agendas, acquires a plan of resolution, and determines needed resources. Policy development results in laws, rules and regulation, ordinances, and policies.

Policy enforcement: Compels others to comply with the laws, rules, regulations, ordinances and policies created in conjunction with policy development.

Note. Source: Minnesota Department of Health, Center for Public Health Nursing. (2001). Public Health Interventions. St. Paul, MN: Author. http://www.health.state.mn.us/divs/opi/cd/phn/wheel.html (accessed 30.01.17) 


\subsection{QoL}

Table 2 identifies the QoL domains and frequencies along with focus areas within each domain that were found in the review of the 23 studies. The number of QoL domains per study ranged from one to six. Table 1 lists the QoL domains specific to each study.

Lawton's Health domain occurred in all studies. Within this domain, biological health occurred most frequently (16 studies). The second and third most frequently appearing domains were Functional Health (20 studies) and Personal Competency (18 studies). For Functional Health, selfmaintenance skills (16 studies), activities of daily living (14 studies), and physical status (8 studies) occurred to a greater extent than other areas. In the Personal Competency domain, specific areas addressed in the studies ranged from one to four, with self-efficacy identified in four studies and educational knowledge identified in three studies. The Psychological Well-Being domain was a focus in 16 studies, with cognitive judgments (18 studies), mental health (9 studies), and self-perception (6 studies) appearing most frequently. The Environment (10 studies) and Social Behavior (9 studies) domains, occurred the least frequently across studies.

Notably, only five of the studies used validated global or health-related quality of life instruments. One study employed a global QoL instrument, and four used health-related quality of life instruments. Further, only two studies utilized age-specific measurements in their interventions. Of those studies incorporating validated instruments, seven studies did not provide any information on validity, with an additional two only citing references.

\subsection{Public health interventions}

Table 3 identifies the level and frequencies of interventions used by nurses in the 23 studies. Although identified interventions occurred in all three levels of practice, 69 interventions were practiced at the individual level compared with 11 interventions each at the community and systems levels. Interventions in the blue and green wedge were used most frequently. Health teaching and counseling often occurred together in a number of the studies. For the red wedge, case finding (individual level) occurred most frequently. Collaboration was the only orange wedge intervention practiced by nurses, but occurred at all three practice levels. Policy development at the systems level was the only intervention used from the yellow wedge. No study interventions were consistent with the following Wheel interventions: disease and other health event investigation, coalition building, community organizing, advocacy, and social marketing. Table 1 identifies the specific Wheel interventions represented in each study. The number of interventions per study ranged from one to six.

\section{Discussion}

\subsection{Perceptions of quality of life}

Although the majority of interventions were focused on the individual level, very few interventions described in this review incorporated older persons' subjective perceptions of QoL or what factors were deemed most important. There are several examples in the literature of research on the meaning of QoL to older persons. In a systematic review, Brown et al. ${ }^{[34]}$ found that good health and being independent and active were very important quality of life components for older persons. In a large sample of over 7000 adults from 22 countries, Molzhan et al. ${ }^{[42]}$ found that having the ability to perform activities of daily living, being healthy, sensory abilities, mobility, freedom, independence, and energy were most important. In a Norwegian study on factors related to QoL among healthy and hospitalized older adults, factors related to cognitive functioning and self-determination were important. ${ }^{[43]}$ In addition, there is evidence that health care providers, as well as significant others, tend to underestimate patients' QoL. Several studies report incongruence between health professionals and patients about the importance of health-related concerns, caring behaviors, pain, and need for information. ${ }^{[44-46]}$

\subsection{Environmental social behavior and QoL domains}

It is notable that fewer studies in the review addressed the social and environmental domains, in comparison to the other four QoL domains. Health, functional health, personal competency, and psychological well-being are more likely to focus on the experiences of individuals. The social and environmental domains will more often involve interactions with social groups, neighborhoods, communities, and organizations, which are consistent with community and system level interventions. With lower frequencies of the social and environmental domains occurring in the group of reviewed studies, it is not surprising community and systems level interventions also occurred less frequently. In addition, many definitions of QoL have not emphasized the environmental domain, which may influence this lack of attention to environmental issues. It is important to integrate the social and environmental domains at all levels of practice.

\subsection{Spiritual and positive aspects of QoL}

None of the interventions included aspects of spirituality, religiousness, or existential issues even though these aspects are evidenced to be particularly important to many older adults' QoL. ${ }^{[47]}$ Spiritual and religious involvement have been shown to buffer stress, reduce depression and cognitive decline, contribute to physical health and healthier lifestyles, 
and reduce healthcare service use. ${ }^{[48]}$ Addressing spiritual concerns is often overlooked in practice, congruent with our findings. ${ }^{[49]}$ For example, in a study involving 4,054 nurses, 95 percent had cared for patients with spiritual needs; however, 92 percent stated they "only occasionally" felt that they could meet these needs. ${ }^{[50]}$ In addition, there very few interventions that focused on older adults' positive emotions. However, positive emotions such as happiness, life satisfaction, fulfillment of expectations, being active, and growth and fulfillment have been shown to contribute to health and longevity among older adults. ${ }^{[51,52]}$ A few interventions, however, were focused on self-efficacy, which includes positive feelings of self-confidence and competence.

\subsection{Culturally relevant interventions for promoting QoL}

Results showed there were no interventions planned from a cross-cultural perspective. Cultural beliefs and values shape people's cognitive representations of themselves and life experiences. Culture is also considered an over-arching form of consciousness influencing people's self-appraisals, attitudes, and values. ${ }^{[53]}$ Three decades ago, Minkler ${ }^{[54]}$ called for culturally relevant ways of thinking about aging, particularly in terms of whether concepts of relevance to aging apply equally across cultures. Conceptual or theoretical formulations could also provide guidance about possible cultural differences in QoL dimensions in terms of their meaning, their relative importance, and how they may co-vary with other factors. A generic QoL instrument, known as the WHOQOL-Bref, has been translated into 40 languages and could be utilized in cross-cultural studies conducted by PHNs. Dragonmirecka et al. ${ }^{[55]}$ used the WHOQOL-Bref instrument in a study that showed cross-cultural differences in physical, psychological, social, and environmental domains among older adults, suggesting the importance of understanding cultural differences in planning interventions.

\subsection{Lack of theoretical perspective}

Almost half of the studies were atheoretical, which has been documented by others for quality of life studies. For example, in a narrative review of 29 empirical studies on QoL among older adults from 2003-2013, findings showed that $90 \%$ of the studies lacked a theoretical or conceptual framework. ${ }^{[56]}$ For those few studies in the review that implemented theoretical perspectives, it was difficult to discern how study variables were connected to the instruments. This difficulty may come from the lack of an existing universal theory of QoL. ${ }^{[32]}$ The finding that the greatest frequency of interventions occurred at the individual level affirms the need for a stronger theoretical base that can guide population-focused nursing practice and research in improving QoL among pop-

Published by Sciedu Press ulations.

Only one population health intervention framework was identified that directly addresses quality of life. The National Advisory Councils on Nurse Education and Practice ${ }^{[57]}$ featured a population health framework ${ }^{[58]}$ in a report on preparing nurses for population health management. This framework identifies health-related quality of life (HRQoL) and mortality as two important population health outcomes. PHNs may find this framework useful for linking interventions and HRQoL for older adult populations. In an analysis of conceptual models for population-focused interventions, including the Public Health Intervention Wheel, only five of the twelve models addressed outcomes. ${ }^{[59]}$ However, linkages between specific interventions and outcomes were missing.

\subsection{Lack of generic, health-related, and age-specific QoL instruments}

Our results showed that researchers applied very few generic or HRQoL instruments in measurement of intervention effectiveness, which raises concern as HRQoL has been identified as an outcome indicator sensitive to healthcare interventions. Possible reasons for limited application of QoL concepts and measurement include nurses' skepticism about its relevance,${ }^{[60]}$ the professional cost of data collection, the nurses' unfamiliarity with the nature of the queries and numerical scale scores, lack of managerial support in providing educational opportunities for learning about the use of validated QoL tools, and lack of education/training for the effective administration of these instruments. ${ }^{[61,62]}$ Our results also showed a lack of the use of age-specific instruments. This finding may be related to the scarcity of age-specific instruments available for QoL assessment. ${ }^{[36]}$ Patrick and Chiang ${ }^{[63]}$ recommended that when assessing QoL instruments, they should be evaluated for their psychometric properties such as reliability, validity, and responsiveness to important clinical change.

\subsection{Scope of public health nursing interventions}

It is not surprising that the most frequent interventions identified in the studies were in the Green and Blue Wedges, since these interventions are consistent with PHN role. Also, the finding that health teaching and counseling often occurred together, is consistent with other studies. ${ }^{[64]}$ The orange wedge intervention of collaboration, which did occur in studies in the review at all three practice levels, fits with the current emphasis on collaborative practice models and inter-professional collaboration. ${ }^{[65]}$ Inter-professional collaboration gives opportunities for crafting interventions for older adults in the community and will be most effective when used at all three practice levels. It is puzzling that 
no advocacy interventions were noted, since nurses serve as the primary contact and advocate for patients, families, and communities and nurses. In the review studies, PHNs were likely advocating for their patients to obtain effective health care, however; advocacy was not emphasized nor evaluated.

The finding that few studies featured interventions at community and systems levels echoes the findings of a recent study on school nurses' use of public health interventions. ${ }^{[64]}$ The National Advisory Council on Nurse Education and Practice $^{[57]}$ calls for a greater emphasis on the population health management role of nurses since they are in a good position to do interventions at all levels of practice. Nurses can bring great value to population health management as public health nursing focuses on assessment and management of all of the physical, biological, social, psychological, and environmental influences on health. ${ }^{[57,66,67]}$ PHNs work is both visible and invisible; they need to promote their work to increase the visibility of their contributions to population health. ${ }^{[68,69]}$

\subsection{Non-experimental, qualitative, and non-research ev- idence}

Expanding the literature search to include non-experimental, qualitative, and non-research evidence may shed light on additional interventions used by PHNs that have the potential to improve QoL. Some nursing interventions at the community and systems level may be difficult to evaluate using experimental research designs. For example, an expert opinion article described a government program developed to promote shared decision-making for wound care with patients. The author delineated a broad array of interventions consistent with the Wheel, including policy development at the systems level. ${ }^{[70]}$

\subsection{Implications for education, practice, policy and re- search}

Nurse educators with clinical expertise is in the care of older adults can enhance student understanding and experience by integrating both QoL concepts and the Public Health Intervention Wheel in teaching nursing students Consideration of the QoL experienced by older adults will assist students in assessing the entire experience of older adults and understanding what factors influence their health, including social and environmental influences. An understanding of both the Public Health Intervention Wheel and QoL concepts gives students the language to describe what they do and plan interventions that aim to improve the QoL of older adults.

In practice, $\mathrm{PHNs}$ can expand their repertoire of interventions to encompass the community and systems levels along with the individual level. PHNs can be more intentional about planning interventions that will promote QoL for older adults. 102
In particular, it is important to consider the views of older adults about what QoL aspects are most important to them. In addition to objective measures of QoL, PHNs need to use subjective appraisals that target older adult views of their QoL. Likewise, governmental and organizational policymakers need to take a more expansive view in order to create policies that address community and systems interventions, particularly those that are planned to contribute to the social and environmental domains of QoL.

More work is needed in designing research that investigates the impact of nurse-led public health interventions on QoL. There is a need to design research that is grounded in QoL theory and models as well as for theory-driven approaches to evaluate how interventions that use individual, community and systems approaches contribute to QoL.

\subsection{Strengths and limitations}

This review uses the concepts of QoL and the Public Health Intervention Wheel to analyze how nurse-led interventions are designed to promote QoL in older adults, which is a unique approach. Although a rigorous search process was implemented to select studies, the limitations of the five-year range and two databases, along with the focus on experimental and quasi-experimental studies may have reduced evidence found on community and systems level nurse-led interventions. In addition, the exclusion of qualitative research may have eliminated studies on older adult perceptions of QoL as well as on older adults' personal beliefs that influence their QoL. Given the inclusion of experimental and quasi-experimental studies, the rigor of the research was high. Also, studies met the majority of quality criteria based on the Johns Hopkins Nursing Evidence-Based Practice Model. An overall weakness of the studies (9 of 23) was the lack of inclusion of reliability and validity data on measures.

\section{Conclusion}

PHNs possess knowledge and expertise to contribute to improved health status for the population of older adults. They are skilled in assessing the whole person in their family and community environments. PHNs who understand strategies that contribute to QoL of older adults and use the Public Health Intervention Wheel to design those strategies at the individual, community, and systems levels of practice have the potential to contribute to improved QoL outcomes for older adults. This review reveals a lack of studies on nurseled interventions that use QoL measures to determine how interventions contribute to QoL for older adults. We call for nurses to implement research designs that utilize QoL measures in addressing population health. QoL measures should emphasize the social and environmental domains of 
QoL. In addition, researchers need to place more emphasis on community and systems interventions and evaluate how they promote QoL The Public Health Intervention Wheel provides a model to guide PHNs in implementing interventions at the community and systems levels along with the individual level of practice.

\section{REFERENCES}

[1] United Nations, Department of Economic and Social Affairs, Population Division. World Population Ageing 2015 (ST/ESA/SER.A/390). 2015. Available from: http://www.un.org/en/development/desa/populat ion/publications/pdf/ageing/WPA2015_Report.pdf (accessed 23.01.17).

[2] United Nations, Department of Economic and Social Affairs, Population Division. World Population Prospects: The 2008 Revision, Highlights, Working Paper No. ESA/P/WP.210. 2009. Available from: http://www.un.org/esa/population/publicat ions/wpp2008/wpp2008_highlights .pdf (accessed 16.12.16).

[3] Organisation for Economic Cooperation and Development (OECD). Policies for Healthy Aging: An Overview. 2009. Available from: http://www.oecd.org/officialdocuments/publicdisplay documentpdf $/$ ?doclanguage $=$ en\&cote $=$ DELSA $/ \mathrm{HEA} / \mathrm{WD} / \mathrm{HWP}$ (2009) 1 (accessed 16.12.16).

[4] Centers for Disease Control and Prevention. Healthy People 2000. Available from: https://www.cdc.gov/nchs/healthy_peopl e/hp2000.htm (accessed 14.12.16).

[5] Centers for Disease Control and Prevention. Healthy People 2010. Available from: https://www.cdc.gov/nchs/healthy_peopl e/hp2010.htm (accessed 14.12.16).

[6] Centers for Disease Control and Prevention. Healthy People 2020. Available from: https://www.cdc.gov/nchs/healthy_peopl e/hp2020.htm (accessed 14.12.16).

[7] European Commission. European Innovation Project on Active and Healthy Ageing. 2016. Available from: http://ec .europa.eu/health/ageing/innovation/index_en.htm (accessed 14.12.16).

[8] Fallowfield L. Quality of Life. The Missing Measurement of Health Care. 1990, Souvenir Press, Human Horizon Series, London.

[9] WHOQOL Group. The World Health Organization's Quality of Life assessment (WHOQOL): position paper from the World Health Organization. Soc Sci Med. 2005; 41: 1403-1409.

[10] WHOQOL-SRPB Group. A cross-cultural study of spirituality, religion, and personal beliefs as components of quality of life. Soc Sci Med. 2006; 62: 1486-1497. PMid:16168541 https://doi.org/ $10.1016 / j$. socscimed.2005.08.001

[11] Kaplan RM, Anderson JP. The general health policy model: An integrated approach. In Quality of Life Assessment in Clinical Trials (Spiker B ed.), Raven Press, New York. 1990.

[12] Jøranson N, Pedersen P, Rokstad AMM, et al. Effects on symptoms of agitation and depression in persons with dementia participating in robot assisted activity: a cluster randomized trial. JAMDA. 2016; 16: 867-873

[13] Lerdal A, Celius EH, Pedersen G. Prescribed exercise: A prospective study of health related quality of life and physical fitness

\section{ACKNOWLEDGeMENTS}

No funds were obtained for completion of this review. Dr. Kalfoss and Dr. Glavin received assistance with the search process through support of the librarian from Diakonova University College in Oslo, Norway.

\section{CONFlicts of InTEREST Disclosure}

The authors declare that there is no conflict of interest. among participants in an officially sponsored municipal training program. J Phys Act Health. 2013; 10: 1016-1023. PMid:23136380 https://doi.org/10.1123/jpah.10.7.1016

[14] Olsen C, Pedersen I, Bergland A, et al. Effect of animal assisted interventions on depression, agitation and quality of life in nursing home residents suffering from cognitive impairment or dementia: a cluster randomized controlled trial. Int J Geriatr Psychiatry. 2016; 31: 1312-1321. http://dx.doi.org.ezproxy. bethel.edu/10 $.1002 /$ gps .4436

[15] Stensvold D, Viken H, Rognmo Ø, et al. A randomized control study of the long term effects of exercise training on mortality in elderly people: study protocol for the Generation 100 study. BMJ Open. 2015; 5: e007519. https://doi.org/10.1136/bmjope n-2014-007519

[16] Stordal E, Bosnes I, Bosnes O, et al. Successful ageing elderly (SAE): a short overview of some important aspects of successful aging. Epidemiologi. 2012; 22: 103-108.

[17] Fassino S, Leombruni P, Daga GA, et al. Quality of life in independent older adults living at home. Arch Gerontol Geriatr. 2002; 35: 9-20. https://doi.org/10.1016/S0167-4943(01) 00210-2

[18] Halvorsrud L, Kirkevold M, Diseth A, et al. Quality of life model: Predictors of quality of life among sick older adults. Res Theory Nurs Pract. 2010; 24: 241-259. PMid:21197919 https ://doi . org/10 $.1891 / 1541-6577.24 .4 .241$

[19] Halvorsrud L, Kalfoss M, Diseth A, et al. Quality of life in older Norwegian adults living at home: a cross-sectional survey. J Res Nurs. 2012; 17: 12-29. https://doi.org/10.1177/17449871 10374273

[20] Hellstrom Y, Hallberg IJ. Perspectives of elderly people receiving home health help on health, care, and quality of life. Health Soc Care Community. 2001; 9: 61-71. PMid:11560722 https: //doi.org/10.1046/j.1365-2524.2001.00282.x

[21] Holland CL, Kellaher L, Peace S, et al. Getting Out and About. In Understanding Quality of Life in Old Age (Walker A ed.). Open University Press, Maidenhead. UK. 2005. 49-63 p.

[22] Netuveli G, Wiggins RD, Hildon Z, et al. Quality of life at older age: evidence from the British Longitudinal Study on Aging (wave 1). J Epidemiol Community Health. 2006; 60: 357-363. PMid:16537355 https://doi.org/10.1136/jech.2005.040071

[23] Netuveli G, Blane DB. Quality of life in older ages. Br Med Bull 2008; 85: 113-126. PMid:18281376 https://doi.org/10.1093/ $\mathrm{bmb} / 1 \mathrm{dn} 003$

[24] Lefort S, Fraser M. Quality of life measurement and its use in the field of learning disabilities. J Learn Disabil. 2002; 6: 22-38. https://doi.org/10.1177/1469004702006003033

[25] Borglin G, Jakobsson U, Edberg AK, et al. Older people in Sweden with various degrees of present quality of life: their health, social support, everyday activities and sense of coherence. Health 
Soc Care Community. 2005; 14: 136-146. PMid:16460363 https : //doi.org/10.1111/j.1365-2524.2006.00603.x

[26] Lee TW, Ko IS, Lee KJ. Health promotion behaviours and quality of life among community-dwelling elderly in Korea: A crosssectional survey. Int J Nurs Stud. 2006; 43: 293-300. PMid:16105668 https://doi.org/10.1016/j.ijnurstu. 2005.06.009

[27] Parse RR. The human becoming modes of inquiry: emerging sciencing. Nurs Sci Q. 2005; 18: 297-330. PMid:16210742 https: //doi.org/10.1177/0894318405380394

[28] Garcia CM, Schaffer MA, Schoon PM. Population-Based Public Health Clinical Manual: The Henry Street Model for Nurses. Sigma Theta Tau International Society of Nursing, Indianapolis, IN. 2014.

[29] Minnesota Department of Health, Center for Public Health Nursing. Public Health Interventions. 2001, St. Paul, MN: Author. Available from: http://www.health.state.mn.us/divs/opi/cd /phn/wheel.html (accessed 30.01.17).

[30] Dearholt SL, Dang D. Johns Hopkins Nursing Evidence-Based Practice. Sigma Theta Tau International Society of Nursing, Indianapolis, IN. 2012. PMid:22970528

[31] Cummins RA. Assessing quality of life for people with disabilities In Quality of Life for People with Disabilities: Models, Research and Practice (Brown R ed.). Stanley Thornes, Cheltenham, UK. 1997. $116-150 \mathrm{p}$.

[32] Bullinger M. Assessing health related quality of life in medicine. An overview over concepts, methods and applications in international research. Restor Neurol Neurosci. 2002. 93-101 p. PMid:12454358

[33] Bowling A. Measuring Disease: A Review of Disease Specific Quality of Life Measurement Scales. Open University Press, Philadelphia. 2001.

[34] Brown J, Bowling A, Flynn T. Models of quality of life: a taxonomy, overview and systematic review of the literature (Project Report). European Forum on Population Ageing Research. 2004. Available from: http://eprints.kingston.ac.uk/17177/ (accessed 0203-16).

[35] Ellingson T, Conn VS. Exercise and quality of life in elderly individuals. J Gerontol Nurs. 2000; 26: 17-25. PMid:11111627 https : //doi.org/10.3928/0098-9134-20000301-06

[36] Haywood KL, Garrat AM, Schmidt LJ, et al. Health Status and Quality of Life in Older People: A Structured Review of Patients-reported Health Instruments. Report from the Patient - reported Health Instruments group (formerly the Patient-Assessed Health Outcomes Programme) to the Department of Health. 2004.

[37] Moons P. Why call it health-related quality of life when you mean perceived health status? Eur J Cardiovasc Nurs. 2004; 3: 275-277. PMid:15572015 https://doi.org/10.1016/j.ejcnurse. 200 4.09.004

[38] Lawton MP. Competence, environmental press, and the adaption of older people. In Aging and Environment. Theoretical Approaches (Lawton MP, Windley PG, Byerts TO ed.). Springer, New York: 1982. 33-50 p.

[39] WHOQOL Group. Development of the WHOQOL: rationale and current status. Int J Ment Health. 1994; 23: 24-26. https://doi org/10.1080/00207411.1994.11449286

[40] Lawton MP. A multidimensional view of quality of life in frail elders. In The concept and measurement of quality of life in the frail elderly (Birren JE, Lubben JE, Rowe, JC, Deutchman DE ed.). Academic Press, San Diego. 1991; 3-37. https ://doi .org/10.1016/B978 -0-12-101275-5.50005-3

[41] Keller LO, Strohschein S, Lia-Hoagberg B, et al. Population-based public health interventions: practice-based and evidence-supported (Part I). Public Health Nurs. 2004; 21: 453-468. PMid:15363026 https://doi.org/10.1111/j.0737-1209.2004.21509.x
[42] Molzhan A, Skevington SM, Kalfoss M, et al. The importance of facets of quality of life to older adults: an international investigation. Qual Life Res. 2010; 19: 293-298.

[43] Kalfoss MH. Issues influencing quality of life among older Norwegian adults: focus Group Results. Res Gerontol Nurs. 2010; 3: 100112. PMid:20415359 https://doi .org/10.3928/19404921-2 0091207-99

[44] Bahrami M, Parker S, Blackman I. Patient's quality of life: a comparison of patient and nurse perceptions. Contemp Nurse. 2008; 29: 67-79. PMid:18844544 https ://doi.org/10.5172/conu. 6 73.29 .1 .67

[45] King A, Hinds PS. Quality of Life from Nurses and Patient Perspectives. Jones and Bartlett Learning, Sudbury, MA. 2011.

[46] Widmark-Pettersson V, Essen L, Sjøden PO. Perceptions of caring among patients with cancer and their staff. Cancer Nurs. 2000; 23: 3239. https://doi.org/10.1097/00002820-200002000-00005

[47] Koening H. Spirituality in Patient Care: Why, How, When and What. Templeton Foundation Press, West Conshohocken, PA. 2007.

[48] Koening H, King D, Carsson VB. Handbook of Religion and Health. Oxford University Press, New York, USA. 2012. PMid:22391469

[49] Molzhan A, Shields L. Why is it so hard to talk about spirituality? Can Nurse. 2008; 104: 25-29.

[50] McSherry W, Jamieson S. An online survey of nurse's perceptions of spirituality and spiritual care. J Clin Nurs. 2011; 20: 1757-1767. PMid:21385257 https://doi.org/10.1111/j.1365-2702.20 $10.03547 . \mathrm{x}$

[51] Fredman L, Hawkes WG, Black S, et al. Elderly patients with hip fracture with positive effect have better functional recovery over two years. J Am Geriatr Soc. 2006; 54: 1074-1081. PMid:16866678 https://doi.org/10.1111/j.1532-5415.2006.00786.x

[52] Kalfoss M. Gender differences in attitudes to ageing among Norwegian older adults. Open Nurs J. 2016; 6: 255-266. https: //doi.org/10.4236/ojn.2016.63026

[53] Oyserman D, Coon HM, Kemmelmeier M. Rethinking individualism and collectivism: evaluation of theoretical assumptions and metaanalysis. Psychol Rev. 2002; 128: 3-72. https://doi.org/10.1 037/0033-2909.128.1.3

[54] Minkler M. Critical perspectives on ageing: new challenges for gerontology. Ageing Soc. 1996; 16: 467-487. https://doi.org/10.1 017/S0144686X00003639

[55] Dragomirecka E, Bartonova J, Eisemann M, et al. Demographic and psychosocial correlates of quality of life in the elderly from a crosscultural perspective. Clin Psychol Psychother. 2008; 15: 193-204. PMid:19115440 https://doi.org/10.1002/cpp. 571

[56] Halvorsrud L, Kalfoss MH. The conceptualization and measurement of quality of life in older adults: a literature review of empirical studies from 1994-2006. Eur J Ageing. 2007; 4: 229-246. https://doi.org/10.1007/s10433-007-0063-3

[57] National Advisory Council on Nurse Education and Practice (NACNEP). Preparing Nurses for New Roles in Population Health Management. Based on the 132nd and 133rd Meetings of the NACNEP 2016 Health Resources and Services Administration (HRSA). 2016. Available from: https://www.hrsa.gov/advisorycommittees/ bhpradvisory/nacnep/Reports/fourteenthreport.pdf (accessed 21.01.17).

[58] Kindig DA, Asada Y, Booske B. A population health framework for setting national and state health goals. JAMA. 2008; 299: 2081-2083. https://doi.org/10.1001/jama.299.17.2081

[59] Bigbee JL, Issel LM. Conceptual models for population-focused public health nursing interventions and outcomes: the state of the art Public Health Nurs. 2012; 29: 370-379. 
[60] Sousa K. Symptom status and health-related quality of life: clinical relevance. JAN. 2003; 42: 571-577. https://doi.org/10.1046/ $\mathrm{j} .1365-2648.2003 .02660 . \mathrm{x}$

[61] Higginson IJ, Carr AJ. Using quality of life measures in the clinical setting. BMJ. 2001; 322: 1297-1300. https://doi.org/10.113 6/bmj. 322.7297.1297

[62] Kalfoss MH. Measuring quality of life among healthy and ill adults. Br J Communit Nurs. 2016; 21(5): 232. PMid:27170407 https: //doi.org/10.12968/bjcn.2016.21.5.232

[63] Patrick DL, Chiang YP. Measurement of health outcomes in treatment effectiveness evaluations: conceptual and methodological challenges. Med Care. 2000; 38: 1114-1125. https://doi .org/10.1097/00 005650-200009002-00005

[64] Schaffer MA, Anderson LJW, Rising S. Public health interventions for school nursing practice. J Sch Nurs. 2016; 32: 195-208. PMid:26404552 https://doi.org/10.1177/10598405156053 61

[65] Sommers LS, Marton KI, Barbaccia JC, et al. Physician, nurse, and social worker collaboration in primary care for chronically ill seniors. Arch Intern Med. 2000; 160: 1825-1833. PMid:10871977 https://doi.org/10.1001/archinte.160.12.1825

[66] Glavin K, Schaffer MA, Halvorsrud L, et al. A comparison of the cornerstones of public health nursing in Norway and in the United States. Public Health Nurs. 2014; 31: 153-166. PMid:24117788 https://doi.org/10.1111/phn.12082

[67] Keller LO, Strohschein S, Schaffer MA. Cornerstones of public health nursing. Public Health Nurs. 2011; 28: 249-260. PMid:21535111 https://doi.org/10.1111/j.1525-1446.2010.00923.x

[68] Clancy A, Svensson T. Perceptions of public health nursing practice by municipal health officials in Norway. Public Health Nurs. 2009; 26: 412-420. PMid:19706124 https://doi.org/10.1111/j.15 25-1446.2009.00799.x

[69] Schaffer MA, Keller LO, Reckinger D. Public health nursing activities: visible or invisible? Public Health Nurs. 2015; 32: 711-720. PMid:25808923 https://doi.org/10.1111/phn.12191

[70] Brown A. Implications of patient shared decision-making on wound care. Wound Care. 2013; Supplement 2: 26-32. https://doi.or g/10.12968/bjcn.2013.18.sup6.s26

[71] Austin J, Hockey D, Williams R, et al. Assessing parental diuretic treatment of decompensated heart failure in the community. $\mathrm{Br}$ J Community Nurs. 2013; 18: 528-534. PMid:24471224 https: //doi.org/10.12968/bjcn.2013.18.11.528

[72] Bakas T, Austin JK, Habermann B, et al. Telephone assessment and skill-building kit for stroke caregivers: a randomized controlled clinical trial. Stroke. 2015; 12: 3478-3487. https ://doi .org/10.116 1/STROKEAHA. 115.011099

[73] Cheng M, Shu-Ling Cheng S, Zhang Q, et al. The effect of continuous nursing intervention guided by chronotherapeutics on ambulatory blood pressure of older hypertensive patients in the community. J Clin Nurs. 2014; 23: 2247-2255. https : //doi .org/10.1111/jo cn. 12502

[74] Cicolini G, Simonetti V, Comparcini D, et al. Efficacy of a nurse-led email reminder program for cardiovascular prevention risk reduction in hypertensive patients: a randomized controlled trial. Int J Nurs Stud. 2014; 51: 833-843.

[75] Duffy JR, Hoskins LM, Dudley-Brown S. Improving outcomes for older adults with heart failure. J Nurs Care Qual. 2010; 25: 56-64. PMid:19512945 https://doi.org/10.1097/NCQ.0b013e3181 adofbd

[76] Friedman B, Li Y, Liebel DV, et al. Effects of a home visiting nurse intervention versus care as usual on individual activities of daily living: a secondary analysis of a randomized controlled trial. BMC
Geriatr. 2014; 14: 24. PMid:24555502 https://doi .org/10.118 6/1471-2318-14-24

[77] Goodman C, Davies C, Fader M, et al. Can district nurses and care home staff improve bowel care for older people using a clinical benchmarking tool? Br J Community Nurs. 2013; 18: 580587. PMid:24335790 https://doi.org/10.12968/bjcn. 2013 .18 .12 .580

[78] Hunger M, Kirchberger I, Holle R, et al. Does nurse-based case management for aged myocardial infarction patients improve risk factors, physical functioning and mental health? The KORINNA trial. Eur J Prev Cardiol. 2015; 22: 442-450. https : //doi .org/10.1177/20 47487314524682

[79] Imhof L, Naef R, Wallhagen MI, et al. Effects of an advanced practice nurse in-home health consultation program for communitydwelling persons aged 80 and older. JAMA. 2012; 6: 2223-2231. https://doi.org/10.1111/jgs.12026

[80] Jansen APD, van Hout HPJ, Nijpels G, et al. Effectiveness of case management among older adults with early symptoms of dementia and their primary informal caregivers: a randomized clinical trial. Int J Nurs Stud. 2011; 48: 933-943. PMid:21356537 https://doi.org/10.1016/j.ijnurstu.2011.02.004

[81] Kerr S, Whyte R, Watson $\mathrm{H}$, et al. A mixed-methods evaluation of the effectiveness of tailored smoking cessation training for healthcare practitioners who work with older people. Worldviews Evid Based Nurs. 2011; 8: 177-186.

[82] Kim GS, Ko S, Lee T, et al. Effects of community-based case management by visiting nurses for low-income patients with hypertension in South Korea. Jpn J Nurs Sc. 2014; 11: 35-43. https: //doi.org/10.1111/j.1742-7924.2012.00229.x

[83] Li Y, Liebel DV, Friedman B. An investigation into which individual activities of daily living are affected by home visiting nurse intervention. Age Ageing. 2013; 42: 27-33. https://doi.org/10.1093/ ageing/afs151

[84] Marquez-Serrano M, Gonzalez-Juarez MPH, Castillo-Castillo LE, et al. Social network analysis to evaluate nursing interventions to improve self-care. Public Health Nurs. 2012; 29: 361-369.

[85] Meethien N, Pothiban L, Ostwald SK, et al. Effectiveness of nutritional education in promoting healthy eating among elders in northeastern Thailand. Pac Rim Int J Nurs Res Thail. 2011; 15: 188-202.

[86] Rodríguez-Cerrillo M, Poza-Montoro A, Fernandez-Diaz E, et al Treatment of elderly patients with uncomplicated diverticulitis, even with comorbidity, at home. Eur J Intern Med. 2013; 24: 430-432. PMid:23623263 https://doi.org/10.1016/j.ejim.2013.03 .016

[87] Rosted E, Poulsen I, Hendriksen C, et al. Testing a two-step nursing intervention focused on decreasing re-hospitalizations and nursing home admission post discharge from acute care. Geriatric Nursing. 2013; 34: 477-485. PMid:24041934 https://doi.org/10.1016/ j.gerinurse.2013.08.001

[88] Rytter L, Jakosen HN, Ronholt F, et al. Comprehensive discharge follow-up in patients' homes by GPs and district nurses of elderly patients. Scand J Prim Health Care. 2010; 28: 146-153. https://doi.org/10.3109/02813431003764466

[89] Shearer NBC, Fleury JD, Belyea M. Randomized control trial of the health empowerment intervention: feasibility and impact. Nurs Res. 2010; 59: 203-211. https ://doi .org/10.1097/NNR. 0b013e31 $81 \mathrm{dbbd} 4 \mathrm{a}$

[90] Søgaard R, Sørensen J, Waldorff FB, et al. Cost analysis of early psychosocial intervention in Alzheimer's Disease. Dement Geriatr Cogn Disord. 2014; 7: 141-153. https://doi .org/10.1159/00 0355368

Published by Sciedu Press 
[91] Wang L, Chen C, Liao W, et al. Evaluating a community-based stroke nursing education and rehabilitation programme for patients with mild stroke. Int J Nurs Pract. 2013; 19: 249-256. PMid:23730856 https://doi.org/10.1111/ijn.12064

[92] Wong FKY, Chow SKY, Chan TMF, et al. Comparison of effects between home visits with telephone calls and telephone calls only for transitional discharge support: a randomized controlled trial. Age Ageing. 2014; 43: 91-97.

[93] Young K, Bunn F, Trivedi D, et al. Nutritional education for community dwelling older people: a systematic review of randomised controlled trials. Int J Nurs Stud. 2011; 48: 751-780. https: //doi.org/10.1016/j.ijnurstu.2011.03.007 